\title{
Gemeinwesenorientierte Primärversorgung (goPV): Eine Herausforderung für Kassenärztliche Vereinigungen
}

SONJA LAAG, UDO

RICHTER, RALPH LÄGEL, UTA HEIDENBLUT UND

SASCHA WOLF

Sonja Laag, Sachgebietsleiterin Versorgungsprogramme, BARMER GEK

Dr. Udo Richter, Geschäftsführer, n:aip Deutschland $\mathrm{GmbH}$

Ralph Lägel, MBA, Manager Contract Development Pain, Pfizer Pharma GmbH

Uta Heidenblut, Vorstandsreferentin, Bundesverband Managed Care e. V.

Dr. Sascha Wolf, Geschäftsführer, Bundesverband $\mathrm{Ma}$ naged Care e. V.

\section{Die soziale Pflegeversicherung ist in ihrer} heutigen Form kaum in der Lage, die anstehenden zukünftigen Versorgungsherausforderungen effizient zu erfüllen.' Ein Kernproblem ist, dass integrierte Versorgungsformen im pflegerischen Bereich auf hohe Hürden stoßen und mit einer flächendeckenden Ausbreitung sektorenübergreifender Behandlungsprozesse zumindest kurzfristig kaum gerechnet werden kann. Deshalb stellt der vorliegende Beitrag als Alternativmodell das Konzept der gemeinwesenorientierten Primärversorgung vor. Ziele sind die Einführung von Care- und Case-Management in die Regelversorgung sowie die Verbindung medizinischer, pflegerischer und sozialer Aspekte zu langfristig angelegten Behandlungsstrategien.

\section{Rahmenbedingungen}

Chronisch kranke, ältere und hochbetagte Menschen bestimmen zunehmend den Versorgungsalltag. Von den im Dezember 2009 rund 2,34 Millionen pflegebedürftigen Personen waren nur 320.000 jünger als 60 Jahre. Noch deutlicher wird dieser Befund, wenn man die altersspezifischen Pflegehäufigkeiten betrachtet. Diese steigen um 0,5 Prozent in jüngeren Jahren auf bis zu 67 Prozent bei über 90-jährigen Frauen (nachfolgend Statistisches Bundesamt). Im Zuge der demographischen Entwicklung wird sich die Anzahl pflegebedürftiger Menschen aller Voraussicht nach verdoppeln.

Der zunehmende Pflegebedarf stellt insbesondere den ambulanten Sektor vor große Herausforderungen. Zwei Drittel der Pflegebedürftigen werden zu Hause versorgt. Davon erhalten 1,07 Millionen ausschließlich Pflegegeld und werden folglich in der Regel durch Angehörige betreut. Weitere 550.000 Pflegebedürftige im privaten Haushalt werden teilweise oder vollständig durch einen ambulanten Pflegedienst versorgt. Seit 2007 ist die Inanspruchnahme ambulanter Dienste um über zehn Prozent gestiegen. Aber auch die Anzahl der in Heimen vollstationär versorgten Personen ist im gleichen Zeitraum auf heute

\footnotetext{
1 Der vorliegende Beitrag basiert auf der Arbeit der interdisziplinär besetzten Projektgruppe »Pflege und Integrierte Versorgung « des Bundesverbands Managed Care e. V. unter der Leitung von Ralph Lägel, MBA, Manager Contract Development Pain, Pfizer Pharma GmbH.
} 
rund 720.000 Personen um knapp fünf Prozent gewachsen. Beide Trends werden sich zukünftig verschärfen.

\subsection{Kostenverschiebung zwischen Pflege- und Krankenkasse}

Obwohl Pflege- und Krankenkasse sich zumeist dasselbe Gebäude teilen und die Mitarbeiter der Pflegekasse zugleich Beschäftigte der Krankenkasse sind, findet eine übergreifende Koordinierung des Leistungsprozesses nicht statt. Die soziale Pflegeversicherung (SPV) unterscheidet sich strukturell von der Gesetzlichen Krankenversicherung (GKV). Dieses betrifft den Umfang der Leistungen (»Teilkasko" in der SPV) wie auch die Wettbewerbsorientierung. Das Image einer Krankenkasse hängt am Beitragssatz bzw. seit Einführung des Gesundheitsfonds an der Zusatzprämie. Dies lässt konkurrierenden Krankenkassen wenig Spielraum für Verträge der Integrierten Versorgung, die sich selten unmittelbar refinanzieren. In der Pflegeversicherung ist der Beitragssatz einheitlich festgesetzt und es erfolgt unter den Pflegekassen für Leistungsaufwendungen und Verwaltungskosten ein Finanzausgleich. Unterschiedliche Vergütungssysteme in GKV und SPV führen zur Kostenkonkurrenz und setzen den falschen Anreiz, Ausgaben zwischen den Versicherungszweigen zu verschieben. Aus dieser Systemlogik resultiert unter anderem, dass sich Maßnahmen der Prävention und Rehabilitation nur dann lohnen, wenn sich deren Erfolg in den Ausgaben der GKV niederschlägt und nicht als externer Effekt in der SPV.

\subsection{Unzureichende Informationen für Pflegebedürftige und Angehörige}

Pflegende Angehörige stellen nach wie vor den größten Teil der häuslichen Versorgung Pflegebedürftiger sicher. Gerade bei chronischen Krankheitsbildern und Multimorbidität mangelt es Pflegebedürftigen und dessen Angehörigen jedoch an ausreichenden Informationen zu den rechtlichen, medizinischen und sozialen Rahmenbedingungen der Pflege und der Koordination unter allen beteiligten Leistungserbringern oder Unterstützern. Mit der Steuerung des Versorgungsprozesses sind die Angehörigen oftmals überfordert. Um solche Situatio- nen zu vermeiden, sollten Konzepte zur Steigerung der Kompetenz und damit der Entlastung der Pflegenden entwickelt werden.

\subsection{Wo ist die Geriatrie?}

Die Multimorbidität setzt einer schematischen, leitlinienbasierten Therapie nach Einzeldiagnosen Grenzen. Geriatrische Kompetenz bedeutet, über eine hohe generalistische Qualifikation zu verfügen. Das oberste Behandlungsziel ist der Erhalt von Selbstständigkeit und Lebensqualität nach Patientenpräferenzen. Da es kaum Forschungsstudien gibt, an denen ältere Menschen beteiligt sind, gibt es zum Umgang mit Multimorbidität auch nur wenig Wissen. Der geriatrische Patient muss vor unkoordinierten und rein spezialisierten Behandlungen geschützt werden. Hierbei spielen mit Blick auf das Behandlungsziel medizinische, pflegerische und soziale Aspekte eine ebenbürtige Rolle (Lübke 2012).

Spezifische geriatrische Versorgungsangebote haben sich bisher nahezu ausschließlich im stationären Versorgungsbereich von Krankenhaus und Rehabilitation entwickelt. Der weitaus größte Teil der gesundheitlichen Versorgung älterer Menschen wird aber im ambulanten Bereich in den Hausarztpraxen geleistet. Den so oft gemalten Horrorszenarien der alternden und vergreisenden Gesellschaft steht eine erstaunliche Gelassenheit in der Entwicklung adäquater Versorgungsstrukturen gegenüber. Wie einst in der Palliativmedizin hängt Deutschland auch hier der Entwicklung europaweit hinterher.

Diese Problematik findet sich auch in den Aus- und Weiterbildungsstrukturen. So existieren nur sieben geriatrische Lehrstühle an 36 medizinischen Fakultäten. Wie ein geriatrieinteressierter Hausarzt an eine Fortbildung kommt, ist nicht so einfach zu beantworten. Ärztekammern, Berufsverbände und andere Organisationen bieten - wenn überhaupt - unterschiedliche Kurse an, die nur Einzelthemen wie Demenz oder das hausärztlich-geriatrische Basisassessment behandeln. Hierbei handelt es sich um die einzige geriatrieorientierte Leistung, die über den Einheitlichen Bewertungsmaßstab (EBM) abrechenbar ist. Aufwand und Morbidität werden zwar auch anhand des Chronikerzuschlags und einer altersadjustierten Versichertenpauschale abgebildet. Ob sie die Arbeit des einzelnen Hausarztes leistungsbezogen vergüten, bleibt jedoch fraglich. Der Hinweis, beim EBM handele es sich im Ganzen um eine Mischkalkulation, tröstet da wenig. Ganz vorne im System der ambulanten vertragsärztlichen Versorgung ist die Gesamtvergütung inzwischen um einen pauschalen Morbiditätsfaktor angepasst. Aber auch hier fühlen Hausärzte, die sich engagiert der Versorgung ihrer alten Patienten widmen, diese finanziellen Zuwendungen nicht unmittelbar, da sie im "großen Waschgang « der Honorarverteilung »weichgespült « werden. Das Konzept der ambulanten geriatrischen Komplexbehandlung bleibt ohne Einbindung in langzeitorientierte Versorgungsstrukturen ein Solitär.

\section{Grenzen der Integrierten Versorgung}

In den letzten Jahren wurden unterschiedliche Versuche unternommen, Disziplinen und Sektoren zu vernetzen. Die größte Hoffnung ruhte hier auf der Integrierten Versorgung (IV, $\mathbb{1 4 0}$ a-d SGB V). Unter den großen Räderwerken der kollektivvertraglichen Systeme hat sich diese aber bislang nicht ausreichend durchgesetzt. Von einer spürbaren und nachhaltigen Ausbreitung integrierter Versorgungskonzepte kann bisher keineswegs gesprochen werden.

Die flächendeckende Umsetzung integrierter Versorgungsverträge scheitert beim Thema Ältere und Pflege gleich aus mehreren Gründen:

- Der geringe Professionalisierungsgrad sowie die ungenügende Marktdurchdringung von Leistungsanbietern. Es gibt nur wenige vernetzte pflegerische Strukturen. »Statt Ausdifferenzierung hat sich in den vergangenen Jahren eine Homogenisierung, also eine Vereinheitlichung, vollzogen. Die Angebotsprofile sind zudem relativ eng und mehr an den Finanzierungsmöglichkeiten als an Bedarfsgesichtspunkten orientiert. Es ist daher nicht verwunderlich, dass sie nicht allen Bedarfslagen älterer Menschen gerecht werden " (Schaeffer/Kuhlmey 2011, S. 182). In der täglichen Praxis sind viele Pflegedienste bereits mit der Realisierung der Standardanforderungen an ihre Bewältigungsgrenzen angelangt. Die IV jedoch erfordert 
die Erfüllung vertraglich vereinbarter Vertrags- und Qualitätsstandards.

- Damit ein Heim IV überhaupt wirtschaftlich betreiben kann, muss ein Großteil der Einrichtung dem Prozess unterworfen werden. Da aber die IV vom Gesetzgeber als Wettbewerbsinstrument der Krankenkassen untereinander gedacht ist, entscheiden die Kassen nach eigenen individuellen Präferenzen (z. B. Anzahl Versicherte), ob sie einen solchen Vertrag abschließen oder nicht. Das bedeutet, dass die Umstellung hausinterner Prozesse nur für eine kleine $Z$ ahl von Pflegebedürftigen erfolgt und damit der Aufwand oftmals in keinem Verhältnis zum betriebswirtschaftlichen Nutzen steht. Oder aber Krankenkassen profitieren als »Trittbrettfahrer", da die internen Abläufe auch für jene Heimbewohner gelten, die bei Kassen versichert sind, die keinen IV-Vertrag mit der Einrichtung haben. Schließlich ist es in der Praxis kaum möglich, eine verbesserte Behandlung anderen Menschen vorzuenthalten.

- Die Vorgabe in $\mathbb{\int} 92$ b Abs. 2 Satz 3 SGB XI, wonach in den Pflegevergütungen keine Aufwendungen berücksichtigt werden, die nicht der Finanzierungszuständigkeit der SPV unterliegen, erschwert die Abstimmung der Vergütung für einen IV-Vertrag.

- Selektivvertragliche Regelungen bedeuten, dass jede Krankenkasse andere Anforderungen und damit auch andere Formulare und Dokumentationspflichten vereinbart. Das bedeutet mehr Bürokratie bei allen Beteiligten.

\section{Bestehende Schwachstellen in der Pflege - Ein Fallbeispiel}

Ein Beispiel aus dem Versorgungsmanagement der Krankenkasse. Ein Mitarbeiter notiert:

89-jährige Frau. Beim Erstkontakt schilderte die alleinstehende Versicherte große Probleme mit einem "Loch im $F u ß$, das ständig blute und von ibr mehr recht als schlecht verpflastert werde. Bei näherem Hinterfragen erklärte die Versicherte, dass sie aufgrund anderer Erkrankungen gezwungen sei, ständig auf dem Rücken zu schlafen. Der Arzt habe ihr bereits zur Vermeidung eines Dekubitus einen sogenannten Fersenschutz verordnet. Dennoch war es offensichtlich zu einem offenen Druckgeschwür gekommen. Durch die blutende Wunde ist die Versicherte nach eigenen Angaben stark eingeschränkt. Sie hatte den Hausarzt gebeten, sich die Wunde im Rabmen eines Hausbesuchs anzusehen. Hierzu war der Arzt nicht bereit. Wie die Versicherte erzählte, habe sie bereits ibre Tasche gepackt, um sich bei weiterer Verschlimmerung ins Krankenhaus fabren zu lassen. Im Verlauf des Gespräches wurde klar, dass die Versicherte für die zukünftige Pflege einen bestimmten Pflegedienst bevorzugt, der vor einigen Jahren den zwischenzeitlich verstorbenen Ehemann betreut hatte. Nach Absprache mit der Frau wurde der Pflegedienst von uns gebeten, kurzfristig eine Beratung für die zukünftigen Pflegeleistungen durchzufübren und sich dabei den Fuß anzuschauen, um bei Bedarf eine fachliche Wundversorgung im Rahmen der Häuslichen Krankenpflege (HKP) einzuleiten. Der Pflegedienst informierte uns, dass die Wundversorgung dringend erforderlich gewesen sei, der Fuß habe bereits gestunken. In der Folgezeit mussten zur Sicherstellung der weiteren HKP noch zablreiche Telefonate geführt werden, weil weder der behandelnde Facharzt noch der Hausarzt die weitere Verordnung der HKP ausstellen wollte."

Dieses Fallbeispiel zeigt eindrücklich, dass die derzeitigen Strukturen in der Pflege nicht überzeugen können. Das Fehlen standardisierter Prozesse, die unzureichende Koordination der Pflegeleistungen, die mangelnde Verzahnung zwischen den Leistungssektoren und das Abschieben von Verantwortlichkeiten führen zu Lücken und Versäumnissen im Behandlungsprozess.

Das Problem ist zu groß und die IV in ihrer heutigen Form zu schwach, als dass sie flächendeckend und nachhaltig Strukturen schaffen könnte, die den Bedürfnissen einer alternden Bevölkerung Rechnung tragen. Die Projektgruppe »Pflege und Integrierte Versorgung « des Bundesverbandes Managed Care e. V. entwickelte aus diesem Grund das Alternativ-Konzept der "gemeinwesenorientierten Primärversorgung « ( $g \mathrm{OPV})$. Eine Ausgestaltung dieses Modells können nur Kassenärztliche Vereinigungen selbst vornehmen. Die skizzierten Ansätze können aber vielleicht Impulse für eine Weiterentwicklung der bestehenden
Strukturen geben.

\section{Die gemeinwesenorientierte Primärversorgung (goPV)}

Bei der goPV handelt es sich um einen alternativen leistungs- und Abrechnungsweg, den eine Kassenärztliche Vereinigung (KV) ihren Hausärzten zum »wie bisher « anbieten kann. Die Idee ist, dass die KV als etablierte Institution teamorientierten Hausärzten eine Weiterentwicklung hin zu Primärversorgerteams ermöglicht. Dafür müsse diese verbindliche Kooperationen mit Pflegediensten eingehen. Aus dem Honorartopf der hausärztlichen Vergütung muss die KV Finanzmittel für die »wie bisher«- Regelversorgung bereitstellen sowie einen zweiten Honorarweg für Hausärzte, die sich freiwillig für den Versorgungsweg goPV entscheiden. Ziel ist es, dass Hausärzte sich geriatrisch qualifizieren und ihre Medizinischen Fachangestellten nach entsprechender Fortbildung (z. B. EVA, VERAH) mit delegationsfähigen Leistungen betrauen sowie mit Pflegeund Sozialdiensten, kommunalen Einrichtungen und regionalen Initiativen verbindliche Kooperationsstrukturen aufbauen und leben. Nur so kann ein Care- und Case-Management etabliert und gelebt werden, das präventive, kurative, rehabilitative und pflegerische Versorgungs- und Unterstützungsleistungen abgestimmt gewährleistet. In der goPV gibt es einen vereinbarten Verantwortlichen, der aus den viel zitierten Schnittstellen Nahtstellen macht.

Die goPV möchte den Beteiligten das Einnehmen einer teamorientierten Haltung ermöglichen. Schwieriger als eine finanzielle Investition scheint nach den Erfahrungen vieler Netzinitiativen (v.a. Arztnetze) die Investition von Vertrauen $\mathrm{zu}$ sein. Sich auf andere Menschen einzulassen, den Aufwand des persönlichen Kennenlernens zu betreiben, gemeinsam am Patienten zu arbeiten und den anderen wertzuschätzen - das alles ist individuell, kostet Überwindung, braucht Zeit und kann nicht angeordnet werden. Es muss aus sich heraus entstehen und ist eine Frage der persönlichen Haltung. Die goPV möchte einen Wettbewerb um Versorger auslösen, die hierzu bereit sind. Ein Wettbewerb, vielleicht auch um ein wenig Demut. 


\subsection{Verankerung im System der Kassenärztlichen Vereinigung}

Im Modell der goPV behält die KV ihre Deutungshoheit über die ambulante vertragsärztliche Versorgung (alte Versorgungsform). Die hausärztliche Versorgung hat in den letzten Jahren eine Aufwertung erfahren. Zum einen durch die 73b-Verträge (Hausarztzentrierte Versorgung, Verpflichtung der Krankenkassen zum Abschluss), zum anderen aber auch durch die öffentliche Diskussion um den (Haus-)Arztmangel. Die Umsetzung der 73b-Verträge erweist sich als schwierig. Versorgungsinhalte werden oft erst im Schiedsverfahren festgesetzt, auch ist das Interesse der Hausärzte bundesweit am Abschluss derartiger Verträge unterschiedlich stark ausgeprägt. Krankenkassen wie Versorgungsforscher kritisieren die wenigen innovativen Ansätze in den 73bVerträgen. Pflege ist eine gesamtgesellschaftliche Aufgabe und bedarf einer starken flächendeckenden strukturellen Verankerung. »Bei einem weitgehenden oder gar totalen Übergang zu selektivvertraglichen Regelungen drohen in stärkerem Maße relevante, insbesondere regionale Differenzen hinsichtlich Qualitätsniveaus der medizinischen Versorgung « (Wille 2010, S. 125).

Durch die »Rebellion« der Hausärzte und die Einführung der IV mag das seit 1931 existierende Monopol der KVen über die ambulante Versorgung etwas ins Wanken geraten zu sein, doch erweist sich die KV bei aller Kritik als »stabil und wertbeständig «. Auch wenn die Ärzte zuweilen ein gespaltenes Verhältnis zu ihren KVen haben, ist der Systemausstieg nicht einmal in Bayern gelungen, der Hochburg der hausärztlichen »Unruhen«. Vielleicht wäre es an der Zeit, die Erfahrungen der letzten Jahre konstruktiv zu nutzen und sich um innovative Versorgungsinhalte und deren ressourcenschonende Umsetzung zu kümmern. Die Ärzteschaft erkämpfte sich Anfang des letzten Jahrhunderts die Gründung von KV und Kammer, um ihre Geschicke selbst in der Hand zu haben. Soll dies so bleiben, muss sie lernen, mit der Pluralität der heutigen Zeit und der ihrer Mitglieder sowie den heutigen komplexen Versorgungsanforderungen differenzierter umzugehen als bisher. Die nach wie vor dominierende Rolle der KBV und KVen ist die des Hono- rarbeschaffers und Honorarverteilers. In der Zukunft werden beide Versorgungsgestaltung insgesamt aktiver betreiben müssen. Öffnung nach außen, Stärke nach innen, könnten die Gebote der Stunde lauten.

Die Wirtschaftsnobelpreisträgerin Elinor Ostrom hat erforscht, wie Menschen in einer Welt, in der es mehr Ansporn zum Konkurrieren statt zum Kooperieren gibt, zusammenarbeiten können. "Dafür brauchen Menschen Ermutigung, Unterstützung und Räume zum Experimentieren. Sie brauchen Institutionen, die es ihnen leicht(er) machen, vor Ort zu kooperieren. Genau hinschauen, Kommunikation von Angesicht zu Angesicht ermöglichen, Vertrauen aufbauen, Regeln und Sanktionen gemeinsam entwickeln, Monitoring ernst nehmen und Ausstiegsmöglichkeiten bieten, wenn die einen kooperieren wollen und die anderen nicht « (Ostrom 2008, S. 17). Die KV ist eine Institution im deutschen Gesundheitswesen, die Kooperation ermöglichen kann.

\subsection{Teilnahme Hausarztpraxen / Pflegedienste / Patienten}

Der goPV-Ansatz wird vor allem von einem Gedanken getragen: Nähe und Vertrauen schaffen. In einer Studie der Universität Witten-Herdecke (2011) äußern befragte Hausärzte, ihre Medizinischen Fachangestellten (MFA) und Pflegedienste immer wieder, wie wichtig es sei, sich vor Ort zu kennen. Initiativen zum besseren Kennenlernen ergreifen sie dennoch nicht. Was ist so schwer daran? Ist das nur eine Frage des Geldes? Kein Gesetzgeber und keine KV kann Vertrauen unter den Akteuren anordnen. Dies muss selbst gewollt und erarbeitet werden und ist eine Grundvoraussetzung für die Teilnahme an der goPV. Der medizinische Bereich muss sich dem pflegerischen öffnen und beide zusammen müssen sich mit dem sozialen Bereich verbünden. Ein Gemeinwesen ist ein soziales Gefüge, in dem Menschen und Systeme etwas verbindet, indem es eine Blickrichtung gibt. Gemeinwesenarbeit aktiviert, nutzt und stärkt infrastrukturelle, soziale und persönliche Ressourcen im regionalen Setting. Teilnehmende Hausärzte und Pflegedienste müssen die Lebensumwelt ihrer Patienten mit im Auge haben. Hier gilt es auch das bislang wenig genutzte
Potenzial der zumeist ortsverbundenen Arzthelferinnen zu nutzen. Da die MFA unmittelbar eingebunden werden, muss die teilnehmende Praxis (Anzahl je nach Größe) über fortgebildete MFA im Sinne der Delegationsvereinbarung (z. B. VERAH, EVA) verfügen. Pflegedienste müssen Personal mit Fortbildung in den Bereichen Care- und Case-Management, Geriatrie oder Palliativpflege vorhalten. Beim Start ist der KV eine Kooperationsvereinbarung zwischen Hausarztpraxis und Pflegedienst vorzuweisen. Hausärzte und Pflegedienste können sich freiwillig für die goPV entscheiden. Sie sollen sich vor Ort suchen und finden und bestmögliche Kooperationsmodelle entwickeln.

Die goPV muss Versicherten aller Krankenkassen zugänglich sein. Ältere und multimorbide Patienten sind keine »souveränen Konsumenten ", die sich eine Versorgung ihrer Wahl aussuchen. Für die Startphase sollte die Zielgruppe genau definiert sein. Die Geriatrie kann hier Ein- und Ausschlusskriterien benennen (Alter, geriatrietypische Multimorbidität, sozialer Befund etc.).

\subsection{Leistungen der goPV}

Die goPV unterteilt sich in einen Arztund einen Koordinationskomplex. Arztkomplex: Der Hausarzt soll »Zeit" für die Behandlung von Patienten haben, die einen erhöhten Versorgungsbedarf aufweisen. Darüber hinaus soll die besondere Fachexpertise (Geriatrie, Palliativmedizin, etc.) gefördert und vergütet werden (geriatrische Assessmentverfahren, Therapieplanung, Monitoring, Hausbesuche etc.). Eine besondere Bedeutung kommt der Beratung und Betreuung der Angehörigen zu.

Koordinationskomplex: Dieses sind Leistungen des Care- und Case-Managements, die nach Absprache zwischen Hausarzt und Pflegedienst entweder von der MFA oder dem Pflegedienst erbracht werden. Der Verantwortliche dabei ist vorab verbindlich festzulegen. Umgangssprachlich ausgedrückt gehören zu diesen Leistungen vor allem auch die »Laufereien «: Abstimmung mit dem Entlassmanagement im Krankenhaus, dem Versorgungsmanagement der Krankenkassen, Apotheken, Sanitätshäuser, Therapeuten, regionalen und sozialen Initiativen, Pflegeheimen. Auch hier spielt die Unterstützung der Angehöri- 
gen eine bedeutende Rolle.

\subsection{Vergütung}

Der goPV-Versorgungsweg wird über eine Komplexpauschale für Arzt- und Koordinationskomplex vergütet. Für den Arztkomplex (fester Europreis) müsste die KV im Rahmen der Honorarverteilung sicherstellen, dass diese als Einzelleistung vergütet wird (je nach Qualifikation Staffelung ab $50 € /$ Quartal/Patient). Für diesen Teil der goPV ist keine Add-on-Finanzierung angedacht (s. Arztkomplex). »Frisches Geld ins System « hingegen braucht es für den Koordinationskomplex. Diese Leistung könnte eine "normale « Leistung der Pflegeversicherung sein und über den Finanzausgleich unter den Pflegekassen ausgeglichen werden. Letztlich ist der Erhalt der Selbständigkeit das Ziel und kommt finanziell vor allem auch der Pflegeversicherung zugute. Eine Beteiligung an der Finanzierung dieser Leistung liegt somit nahe. Im Rahmen der anstehenden Pflegereform könnte dieses ein kleiner Schritt zur finanziellen und leistungsbezogenen Verzahnung der Sektoren sein.

\section{Arztkomplex:}

Der ärztliche Vergütungskomplex sollte nach Qualifikation des Hausarztes gestaffelt sein (z. B. um Anreize für geriatrische und palliativmedizinische Fortbildung zu geben). Bereits bestehende EBM-Leistungen könnten zu einer Pauschale gebündelt werden, für die dann ein Abrechnungsausschluss besteht:

- Versichertenpauschale (ab 60. Lj.)

- Chroniker-Zuschlag

- (evtl. Hausbesuch)

- Präventions-Check

- Delegationszuschlag für Medizinische Fachangestellte

- hausärztlich-geriatrisches Basisassessment

- ggf. Qualitätszuschlag (z. B. für besondere Demenzdiagnostik, Palliativoder Schmerzweiterbildung usw.)

- DMP-Programmpauschale

- (evtl. Vergütungen aus Verträgen der Spezialisierten Ambulanten Palliativversorgung (SAPV)

\section{Koordinationskomplex:}

Übernimmt der Pflegedienst die Koordinationsleistung, so kann dieser den Koordinationskomplex über die KV abrechnen (alle ureigensten Leistungen des Pflegedienstes haben mit der goPV nichts zu tun und werden weiter wie bisher erbracht und abgerechnet). Übernimmt die Arztpraxis (MFA) die Koordination, so wird diese dem Arzt mit seinem Arztvergütungskomplex vergütet. Zur Vergütungshöhe des Koordinationskomplexes kann an dieser Stelle keine Orientierung gegeben werden.

\section{Zusammenfassung}

Bei der goPV handelt es sich um ein Gedankenmodell. Besonders die honorartechnischen Fragen können hier nur grob skizziert werden. Die Welt der ambulanten vertragsärztlichen Vergütung ist komplex. Wie groß die Spielräume innerhalb der KVen unter Berücksichtigung von Rahmenvorgaben durch die Kassenärztliche Bundesvereinigung sind und wie sie sich dann im Verhältnis zu den Vorstellungen der Krankenkassen verhalten, kann sich nur bei konkreter Umsetzung zeigen. Mit dem Versorgungsstrukturgesetz stehen die Zeichen auf Re-Regionalisierung.

Die goPV hat sich Erkenntnisse aus den neuen Versorgungsformen zunutze gemacht. DMP, IV, SAPV formulieren Qualitätsanforderungen, orientieren sich verbindlich an Behandlungspfaden, sind interdisziplinär und multiprofessionell im Teamansatz ausgerichtet und wollen Sektoren überwinden. Bei allen Schwierigkeiten hat besonders die SAPV gezeigt, dass sich Arzt und Pflege kennenlernen, vertrauen und schätzen können. Die goPV könnte auch ein Lösungsweg für die noch offene Frage einer flächendeckenden Umsetzung der Allgemeinen Ambulanten Palliativversorgung (AAPV) sein. Ohne die starken institutionellen Arme der Kollektivvertragspartner kann eine flächendeckende Vernetzung kein Standard werden.

\section{Fazit}

Integrierte Versorgungsformen haben sich in der Pflege bislang nicht flächendeckend durchsetzen können. Umso wichtiger ist es, alternative Konzepte zu entwickeln, um eine ganzheitliche Versorgung alter, multimorbider und pflegebedürftiger Menschen sicherzustellen. Ein möglicher Gesamtansatz stellt das von der Projektgruppe »Pflege und Integrierte Versorgung" des Bundesverbands Managed Care e. V. entwickelte Konzept der gemeinwesenorientierten Primärversorgung (goPV) dar. Durch die Etablierung kooperativer Strukturen, die konsequente Anwendung von Care- und Case-Management sowie die dadurch ermöglichte Förderung des Selbstmanagements und der Kompetenz von pflegenden Angehörigen trägt dieser Ansatz den wesentlichen Herausforderungen einer immer älter werdenden Gesellschaft Rechnung.

Die Entscheidung für die Teilnahme an der $g o P V$ ist für den Leistungserbringer wie Patienten freiwillig. Sollten Kooperationen nicht zustande kommen oder sich Erwartungen nicht erfüllen, wird weiterhin über die normale Regelversorgung versorgt. Somit bietet die goPV die Chance eines sanften und risikofreien Einstiegs für Arztpraxen und Pflege in innovative Arbeitsstrukturen. Der Gesetzgeber will die Primärversorgung stärken und die nicht-ärztlichen Berufe - hier besonders die Pflege - fördern, um eine abgestimmte und kontinuierliche Versorgung älterer und vulnerabler Menschen zu gewährleisten. Neben eigenen Anstrengungen liegt die Zukunft der nicht-ärztlichen Berufe auch in den Händen der Ärzte. Mit Blick auf die Geschichte der Profession war es die Ärzteschaft, die das Wohl und Weh der nicht-ärztlichen Berufe bestimmt und diese in Deutschland zu einem »Dienst am Arzt« und danach erst zu einem Dienst am Patienten gemacht hat. Die goVP möchte gerade innerhalb der Ärzteschaft jene Leistungserbringer gewinnen, die ein multiprofessionelles Arbeitsverständnis haben. Die Sorgefähigkeit der Gesellschaft zu erhalten, so, wie es der aktuelle Altenbericht fordert, gelingt nur durch Kooperation, Vertrauen und Wertschätzung unter den Professionellen und unter Einbeziehung der sogenannten Laien. Um es mit Alfred Polgar zu sagen: »Euer Herz, mein Lieber, schlägt den nackten Rhythmus, wo aber bleibt die Melodie? 


\section{Literatur}

Bundesministerium für Familie, Senioren Frauen und Jugend (2010): Sechster Bericht zur Lage der älteren Generation in der Bundesrepublik Deutschland. Berlin.

Bundesverband Geriatrie e. V. (2010): Weißbuch Geriatrie, 2. Auflage. Stuttgart: Kohlhammer

Döhler, M. (1997): Die Regulierung von Professionsgrenzen. Struktur und Entwicklungsdynamik von Gesundheitsberufen im internationalen Vergleich. Frankfurt: Campus Verlag.

Gensichen, J., Rosemann, T. (2007): Das Chronic-Care-Modell: Elemente einer umfassenden Behandlung für Patienten mit chronischen Krankheiten. In: Zeitschrift für Allgemeinmedizin, Nr. 83, S. 483-486.

Huerkamp, C. (1985): Der Aufstieg der Ärzte im 19. Jahrhundert. Göttingen: Vandenhoeck \& Ruprecht

Kuhlmey, A. (2009): Spezielle Versorgungsanforderungen bei älteren und alten Menschen. In: Zeitschrift für Gerontologie und Geriatrie, 42(6), S. 425-431.

Lübke, N., Ziegert, S., Meinck, M. (2008): Geriatrie. Erheblicher Nachholbedarf in der Weiter- und Fortbildung. In: Deutsches Ärzteblatt, Jg. 105, Heft 21.

Lübke, N. (2012): Brauchen alte Menschen eine andere Medizin? Medizinische Einordnung spezieller Behandlungserfordernisse älterer Menschen. In: Versorgungs-Report 2012, Stuttgart: Schattauer

Ostrom, E. (2008): Was mehr wird, wenn wir teilen. Vom gesellschaftlichen Wert der Gemeingüter. München: oekom Verlag.

Schaeffer, D., Kuhlmey, A. (2011): Neue Modelle für die pflegerische Versorgung alter Menschen - Herausforderungen in der ambulanten Pflege. In: Versorgungs-Report 2012, Stuttgart: Schattauer.

Schmacke, N. (2011): Alter und Krankheit: eine Frage neuer Versorgungsformen, nicht nur für alte Menschen. In: VersorgungsReport 2012, Stuttgart: Schattauer.

Sachverständigenrat zur Begutachtung der Entwicklung im Gesundheitswesen (2007): Kooperation und Verantwortung - Voraussetzungen einer zielorientierten Gesundheitsversorgung, Baden-Baden: Nomos.

Statistisches Bundesamt (2011): Pflegestatistik 2009, Wiesbaden: Statistisches Bundesamt.

Universität Witten-Herdecke (2011): ZuVerSicht. Die Zukunft der hausärztlichen Versorgung aus gesundheitsberuflicher und Patienten-Sicht.

Wille, Eberhard (2010): Stärkung der Wettbewerbsorientierung durch Ausweitung der selektivvertraglich organisierten Gesundheitsversorgung. In: Effizientere und leistungsfähigere Gesundheitsversorgung als Beitrag für eine tragfähige Finanzpolitik in Deutschland. IGES (Hrsg.). Baden-Baden.

\section{Handbuch zum gesamten Sozialrecht}

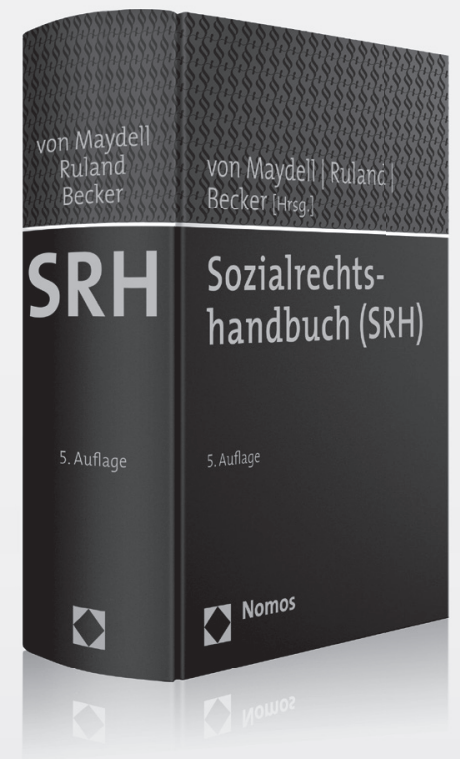

Wie kaum ein anderes Rechtsgebiet spiegelt das Sozialrecht die rechtspolitische Diskussion wider. Konsequenz ist eine Fülle unterschiedlichster Rechtsentwicklungen in den besonderen Teilen des Sozialrechts, die das Sozialrechtshandbuch systematisiert, verknüpft und übersichtlich in einem Band darstellt.

Die Neuauflage bringt das SRH auf den aktuellen Stand der Gesetzgebung und Rechtsprechung.

\section{Weitere Informationen: www.nomos-shop.de/13448}

\section{Sozialrechtshandbuch (SRH)}

Herausgegeben von Prof. Dr. Bernd Baron von Maydell, Prof. Dr. Franz Ruland, Geschäftsführer des Verbandes Deutscher Rentenversicherungsträger (VDR) i.R. und Prof. Dr. Ulrich Becker, LL.M.

5. Auflage 2012, ca. $1.600 \mathrm{~S}$., geb., ca. 148,- $€$

ISBN 978-3-8329-6462-7

Erscheint ca. Mai 2012

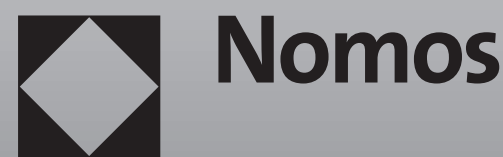

